\section{Nuclear Fusion and Non-Fusion in Theobroma cacao L.}

Cytological investigations carried out on Theobroma cacao have revealed the fact that the incompatibility reaction in this species is based on non-fusion between male gametes and female haploid nuclei carrying the same dominant $S$ allele $\mathbf{1}$. Incompatibility pollinated cacao ovaries contain approximately 25, 50 or 100 per cent of ovules wherein fusions between the first male gamete and the egg nucleus and between the second male gamete and the polar nuclei have not taken place. In such ovules, the egg and male nuclei appear always to lie in actual contact, each preserving its spherical shape; the second male gamete and the polar nuclei, however, do not show such regularity of behaviour, for the two polar nuclei may sometimes become dissociated from one another, the male nucleus lying elsewhere in the embryo-sac or in contact with one of the separated polar nuclei.

When fusion does take place between a male gamete and the polar nuclei, the male gamete invariably places itself at some point on the line of juncture of the two polar nuclei, so that the male nucleus lies in simultaneous contact with both female nuclei before fusion takes place. The male gamete appears not to fuse at random over the surface of one or other of the polar nuclei but always comes into the position where all three nuclei are in mutual contact. The male nucleus then insinuates itself between the polar nuclei, assuming a more and more fusiform shape as it penetrates. Finally, the contents of the male nucleus mingle with the nucleoplasm of one of the polar nuclei. The process is shown in diagrammatic form in Fig. 1.
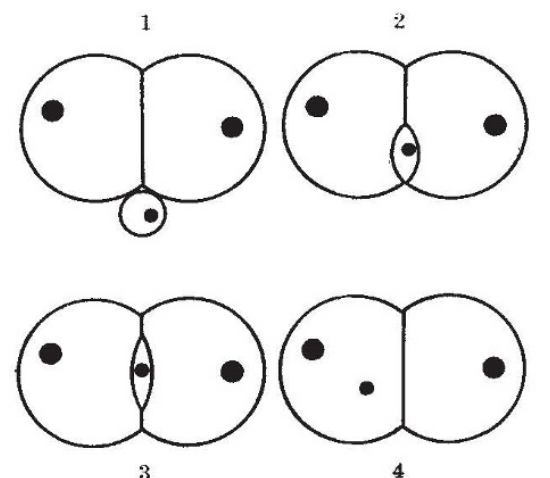

4

Fig. 1. Four stages in the fusion of a male gamete with the polar nuclei in cacao (diagrammatic)

This fusion behaviour can be likened to the coalescence of oil droplets in an extremely unstable oil/ water emulsion; here the fusing oil droplets tend to share large areas of their surfaces and hence assume a polyhedral form ${ }^{2}$. The male nucleus, originally spherical (as is a single oil droplet), arranges itself between the polar nuclei in such a manner that its entire surface is shared with these two nuclei. Fusion with one or other polar nucleus is a purely random process.

In the case of non-fusing nuclei, entirely different interfacial conditions must exist and the system can be likened to a stable oil/water emulsion in which no coalescence of the oil droplets takes place. In emulsions stabilized, for example, with a protein, direct oil-to-oil contact between droplets is prevented by the presence of a monolayer of adsorbed protein at the surface of each droplet. This condition does not imply that the stabilized droplets cannot make contact, but rather that when contact is made they preserve their spherical form. Clusters of spherical oil droplets can be seen under the mieroscope in many emulsions.

The not infrequent separation of the two polar nuclei in ovules showing non-fusion leads us to suggest that this effect is brought about by the presence of a strongly but specifically adsorbed substance in the cytoplasm of the male nucleus carrying the dominant $S$ allele. When both male and polar nuclei carry the same dominant allele (for example, $S_{1}$ nuclei in in $S_{1 \cdot 2}$ selfed or in $S_{1 \cdot 2} \times S_{1 \cdot 3}$ ), the substance which 'interferes' with nuclear fusion diffuses from the male cytoplasm into that associated with the polar nuclei, from which it interacts with specific sites on the surface of both polar nuclei, leading to protection against fusion (or stabilization of all three bodies). In these circumstances the separation of the nuclei is understandable.

No specific adsorption sites, apparently, are present at the surface of polar nuclei which carry the recessive allele (for example, $S_{2}$ nuclei in the $S_{1 \cdot 2}$ genotype) for fusion with any male nucleus will always take place. When the polar nuclei carry a dominant $S$ allele different from that of the male gamete, fusion again will ensue; for example, $S_{3}$ polar nuclei in an $S_{3.4}$ genotype will fuse with $S_{1}$ male gametes derived from an $S_{1.2}$ genotype because the $S_{3}$ sites are not specific adsorption sites for the $S_{1}$ protective substance. If the male nucleus does not carry a dominant $S$ allele, no protective substance is present in its cytoplasm; it will therefore always fuse with any female nuclei.

In view of the specificity of the non-fusion reaction, the protective substance is likely to be proteinaceous in character.

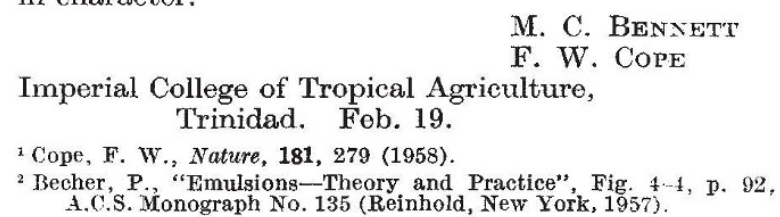

\section{Genetics of Dieldrin Resistance in Lucilia cuprina Wied.}

IN a comprehensive paper on insecticidal resistance in anopheline mosquitoes Davidson ${ }^{1}$ demonstrated that resistance to dieldrin in Anopheles gambiae is due to a single gene, partially dominant. In this work the discriminating dose technique, supported by $\mathrm{Milani}^{2}$, for genetical studies on insecticidal resistance in insects was utilized.

Employing this method in a study of the genetics of dieldrin resistance in Lucilia cuprina, in which insecticidal resistance has been demonstrated ${ }^{3}$, it is indicated that dieldrin resistance in this insect is also due to a partially dominant single gene. To date information is available only from a cross between susceptible female flies and a single resistant male fly. The results, however, conform so elosely to a Mendelian interpretation on a single gene basis that it can be expected that additional work will merely confirm the above conclusion.

In this preliminary study a single resistant male fly, which survived $4 \mu \mathrm{gm}$. of dieldrin, was crossed with ten susceptible female flies. The male fly was taken from strain of flies undergoing selection in an 